\title{
Valid Sequents in Many-Valued Logics
}

\author{
Mitio Takano \\ Niigata University
}

In this paper, we shall be concerned with many-valued (propositional) logics with designated values.

For each of the above-mentioned logics, we shall define the notion of validity of sequents so that a sequent is valid iff for every valuation of (propositional) variables the truth-values of some formulas occurring in the succedent of the sequent are designated whenever so are those of all formulas occurring in the antecedent. Then, we shall characterize those sets which are exactly the sets of all the valid sequents in some logics. Also, we shall investigate the relationship between the set of all the valid sequents of one logic and that of another, or between the set of all the valid sequents and the number of truth-values of a logic, principally of a finite one. In the study of the latter subject, the notion of regularity of logics will play the leading role.

In this article, when $S$ is a set $\operatorname{Card}(S)$ shall denote the cardinality of $S$, and each non-negative integer shall be identified with the corresponding finite cardinal.

The author wishes to express his hearty gratitude to Prof. Kokio Shirai for his help during the preparation of this paper.

\section{§1. Syntactical preliminaries}

1.1. Primitive symbols of the language with which we shall be concerned throughout this paper are:

1) countably many (propositional) variables,

2) at most countably many (propositional) connectives each with its own finite arity, and

3) parentheses, comma and arrow.

1.2. Formulas are defined by the following recursion:

(i) A variable is a formula.

(ii) If $F$ is a $k$-ary connective and $A_{1}, \cdots, A_{k}$ are formulas, then $F\left(A_{1}, \cdots, A_{k}\right)$ is also a formula.

(iii) Formulas are obtained only under the clauses (i) and (ii).

There are countably many formulas.

1.3. A sequent is an expression of the form

$$
\begin{gathered}
\sigma: A_{1}, \cdots, A_{l} \rightarrow B_{1}, \cdots, B_{m}, \\
-245-
\end{gathered}
$$


where $l, m$ and $A_{1}, \cdots, A_{l}, B_{1}, \cdots, B_{m}$ are arbitrary non-negative integers and formulas, respectively. Of the sequent $\sigma, A_{1}, \cdots, A_{l}$ and $B_{1}, \cdots, B_{m}$ form the antecedent and succedent, respectively.

There are countably many sequents.

\section{§ 2. Many-valued logics}

2.1. We shall define the notion of (many-valued) logics and some related ones.

2.11. A logic is a triplet $\mathscr{L}=(T, \Phi, D)$ with the following properties:

L1. $T$ is a non-empty set.

L2. $\Phi$ is such a function whose domain is the set of all the connectives that $\Phi(F)$ is a function mapping $T^{k}$ into $T$ for each $k$-ary connective $F$.

L3. $D$ is a subset of $T$.

$T$ is the set of the truth-values, $\Phi(F)$ the truth-function of $F$ for each connective $F$, and $D$ the set of the designated values of $\mathscr{L}$.

By $\operatorname{Card}(\mathscr{L})$ we mean $\operatorname{Card}(T)$, and $\mathscr{L}$ is finite iff so is $\operatorname{Card}(T)$.

A pair $(T, \Phi)$ satisfying $\mathrm{Ll}$ and L2 above is called a pre-logic.

2.12. Let $\mathscr{L}=(T, \Phi, D)$ and $\mathfrak{M}=(U, \Psi, E)$ be logics.

A function $h$ mapping $T$ into $U$ with the following properties $\mathrm{H} 1$ and $\mathrm{H} 2$ is a homomorphism of $\mathscr{L}$ into $\mathfrak{M}$ :

H1. $h\left(\Phi(F)\left(t_{1}, \cdots, t_{k}\right)\right)=\Psi(F)\left(h\left(t_{1}\right), \cdots, h\left(t_{k}\right)\right)$, for every $t_{1}, \cdots, t_{k} \in T$ and every $k$-ary connective $F$.

H2. $h(t) \in E \Leftrightarrow t \in D$, for every $t \in T$.

A homomorphism of $\mathscr{L}$ into $\mathfrak{M}$ is surjective or bijective iff it maps $T$ into $U$ surjectively or bijectively, respectively.

2.13. Let $\mathscr{L}=(T, \Phi, D)$ be a logic.

A binary relation $\equiv$ on $T$ is a congruence relation on $\mathscr{L}$ iff the following properties hold:

C1. $\equiv$ is an equivalence relation on $T$.

C2. $\Phi(F)\left(s_{1}, \cdots, s_{k}\right) \equiv \Phi(F)\left(t_{1} \cdots, t_{k}\right)$ whenever $s_{1} \equiv t_{1}, \cdots, s_{k} \equiv t_{k}$, for every $k$ ary connective $F$.

C3. $s \in D \Leftrightarrow t \in D$ whenever $s \equiv t$.

A non-empty subset $S$ of $T$ is an $\mathscr{L}$-subset iff $\Phi(F)\left(t_{1}, \cdots, t_{k}\right) \in S$ whenever $t_{1}, \cdots$, $t_{k} \in S$ for every $k$-ary connective $F$.

An $\mathscr{L}$-subset is proper iff it is a proper subset of $T$.

2.2. Let be given a pre-logic $(T, \Phi)$. Suppose that $A\left(p_{1}, \cdots, p_{n}\right)$ is a formula containing only the distinct variables $p_{1}, \cdots, p_{n}$, and that $t_{1}, \cdots, t_{n}$ are elements of $T$. We shall define the truth-value, which is denoted by $A^{\Phi}\left[t_{1}, \cdots, t_{n}\right]$, of $A\left(p_{1}, \cdots\right.$, $\left.p_{n}\right)$ at $\left(t_{1}, \cdots, t_{n} / p_{1}, \cdots, p_{n}\right)$ in $(T, \Phi)$ as follows: 
(i) If $A\left(p_{1}, \cdots, p_{n}\right)$ is $p_{i}$, then

$$
A^{\Phi}\left[t_{1}, \cdots, t_{n}\right]=t_{i}
$$

(ii) If $A\left(p_{1}, \cdots, p_{n}\right)$ is $F\left(A_{1}\left(p_{1}, \cdots, p_{n}\right), \cdots, A_{k}\left(p_{1}, \cdots, p_{n}\right)\right)$, then

$$
A^{\Phi}\left[t_{1}, \cdots, t_{n}\right]=\Phi(F)\left(A_{1}^{\Phi}\left[t_{1}, \cdots, t_{n}\right], \cdots, A_{k}{ }^{\Phi}\left[t_{1}, \cdots, t_{n}\right]\right) \text {. }
$$

The truth-value $A^{\Phi}\left[t_{1}, \cdots, t_{n}\right]$ depends only on those $t_{i}$ 's and $\Phi(F)$ 's for which $p_{i}$ and $F$ actually occur in $A\left(p_{1}, \cdots, p_{n}\right)$.

Now, we shall state a proposition which deals with the behavior of the truthvalue under the substitution of a formula for a variable. The proof is carried out by induction on the complexity of formulas, and we omit it.

Proposition 1. Let $(T, \Phi)$ be a pre-logic. Suppose that $p, q_{1} \cdots, q_{n}$ are distinct variables, $A\left(p, q_{1}, \cdots, q_{n}\right)$ and $B\left(q_{1}, \cdots, q_{n}\right)$ formulas containing only the variables shown, and that $C\left(q_{1}, \cdots, q_{n}\right)$ is the result $A\left(B\left(q_{1}, \cdots, q_{n}\right), q_{1}, \cdots, q_{n}\right)$ by the substitution of $B\left(q_{1}, \cdots, q_{n}\right)$ for $p$ throughout $A\left(p, q_{1}, \cdots, q_{n}\right)$. Then for every $t_{1}, \cdots, t_{n} \in T$,

$$
C^{\Phi}\left[t_{1}, \cdots, t_{n}\right]=A^{\Phi}\left[B^{\Phi}\left[t_{1}, \cdots, t_{n}\right], t_{1}, \cdots, t_{n}\right] .
$$

2.3. Let be given a logic $\mathscr{L}=(T, \Phi, D)$, and suppose that $\sigma\left(p_{1}, \cdots, p_{n}\right)$ is a sequent containing only the distinct variables $p_{\mathbf{1}}, \cdots, p_{*}$.

Let $t_{1}, \cdots, t_{n}$ be elements of $T$. Then, $\sigma\left(p_{1}, \cdots, p_{n}\right)$ is true at $\left(t_{1}, \cdots, t_{n} / p_{1}, \cdots\right.$, $\left.p_{n}\right)$ in $\mathscr{L}, \sigma\left[t_{1}, \cdots, t_{n}\right]$ is true in $\mathscr{L}$ for short, iff either the truth-values at $\left(t_{1}, \cdots, t_{n} / p_{1}\right.$, $\left.\cdots, p_{n}\right)$ in $(T, \Phi)$ of some formulas occurring in the antecedent of $\sigma\left(p_{1}, \cdots, p_{n}\right)$ do not belong to $D$ or those of some formulas occurring in the succedent do. $\sigma\left(p_{1}, \cdots, p_{n}\right)$ is false at $\left(t_{1}, \cdots, t_{n} / p_{1}, \cdots, p_{n}\right)$ in $\mathscr{L}, \sigma\left[t_{1}, \cdots, t_{n}\right]$ is false in $\mathscr{L}$ for short, iff it is not true there.

$\sigma\left(p_{1}, \cdots, p_{n}\right)$ is valid in $\mathscr{L}$ iff $\sigma\left[t_{1}, \cdots, t_{n}\right]$ is true there for every elements $t_{1}, \cdots$, $t_{n}$ of $T$. We shall denote the set of all the valid sequents in $\mathscr{L}$ by $W(\mathscr{L})$.

Now, we shall show a useful lemma, $2^{\circ}$ ) of which is essentially due to Tarski [3] in which valid formulas and only bijective homomorphisms are concerned.

Lemma 2. Let $\mathscr{L}$ and $\mathfrak{M}$ be logics. Then:

$\left.1^{\circ}\right)$ If there is a homomorphism of $\mathscr{L}$ into $\mathfrak{M}$, then

$$
W(\mathfrak{D}) \subseteq W(\mathscr{L})
$$

$\left.2^{\circ}\right)$ If there is a surjective homomorphism of $\mathscr{L}$ into $\mathfrak{M}$, then

$$
W(\mathfrak{M})=W(\mathscr{L}) .
$$

For the proof of Lemma 2 we shall state Proposition $3,1^{\circ}$ ) of which is obtained by induction on the complexity of formulas, while $2^{\circ}$ ) of which readily follows from $1^{\circ}$ ). It is easy to deduce Lemma 2 from Proposition 3.

Proposition 3. Let $\mathscr{L}=(T, \Phi, D)$ and $\mathfrak{M}=(U, \Psi, E)$ be logics and $h$ a function mapping $T$ into $U$. Assume that $p_{1}, \cdots, p_{n}$ are distinct variables. Then: 
$\left.1^{\circ}\right)$ If $h$ satisfies $\mathrm{H1}$, then for every $t_{1}, \cdots, t_{n} \in T$ and every formula $A\left(p_{1}, \cdots, p_{n}\right)$ containing only the variables shown,

$$
h\left(A^{\Phi}\left[t_{1}, \cdots, t_{n}\right]\right)=A^{T}\left[h\left(t_{1}\right), \cdots, h\left(t_{n}\right)\right] .
$$

$\left.2^{\circ}\right)$ If $h$ is a homomorphism of $\mathscr{L}$ into $\mathfrak{M}$, then for every $t_{1}, \cdots, t_{n} \in T$ and every sequent $\sigma\left(p_{1}, \cdots, p_{n}\right)$ containing only the variables shown,

$$
\sigma\left[t_{1}, \cdots, t_{n}\right] \text { is true in } \mathscr{L} \Leftrightarrow \sigma\left[h\left(t_{1}\right), \cdots, h\left(t_{n}\right)\right] \text { is true in } \mathfrak{M} \text {. }
$$

2.4. In this item, we shall construct a logic from a set of formulas.

Let be given a set $D$ of formulas. We let $\mathscr{L}(D)$ be the logic $(T, \Phi, D)$ defined by:

(a) $T$ is the set of all the formulas.

(b) $\Phi(F)\left(C_{1}, \cdots, C_{k}\right)=F^{\prime}\left(C_{1}, \cdots, C_{k}\right)$, for every formulas $C_{1}, \cdots, C_{k}$ and every $k$-ary connective $F$.

(c) $D=D$.

Then we easily obtain the following

Proposition 4. Let $D$ be a set of formulas and $\mathscr{L}(D)=(T, \Phi, D)$ be as above. Assume that $p_{1}, \cdots, p_{n}$ are distinct variables. Then:

$\left.1^{\circ}\right)$ Let be given a formula $A\left(p_{1}, \cdots, p_{n}\right)$ containing only the variables shown. Then for every formulas $C_{1}, \cdots, C_{n}, A^{\Phi}\left[C_{1}, \cdots, C_{n}\right]$ is the result $A\left(C_{1}, \cdots, C_{n}\right)$ of substitution of $C_{1}, \cdots, C_{n}$ for $p_{1}, \cdots, p_{n}$ throughout $A\left(p_{1}, \cdots, p_{n}\right)$.

$\left.2^{\circ}\right)$ Let be given a sequent $\sigma\left(p_{1}, \cdots, p_{n}\right)$ containing only the variables shown. Then $\sigma\left(p_{1}, \cdots, p_{n}\right)$ belongs to $W(\mathscr{L}(D))$ iff for every formulas $C_{1}, \cdots, C_{n}$ some formulas occurring in the succedent of the sequent $\sigma\left(C_{1}, \cdots, C_{n}\right)$ belong to $D$ whenever all occurring in the antecedent do, where $\sigma\left(C_{1}, \cdots, C_{n}\right)$ is the result of substitution of $C_{1}$, $\cdots, C_{n}$ for $p_{1}, \cdots, p_{n}$ throughout $\sigma\left(p_{1}, \cdots, p_{n}\right)$.

2.5. In this last item of $\S 2$, we shall show three ways of construction of another logic from one.

2.51. Let $\mathscr{L}=(T, \Phi, D)$ be a logic and $\equiv$ a congruence relation on it.

We let $\mathscr{L} \mid \equiv$ be the logic $(U, \Psi, E)$ defined by (a), (b) and (c) below. For each $t \in T$, we let $t / \equiv$ be the equivalence class to which $t$ belongs.

(a) $U=\{t|\equiv| t \in T\}$.

(b) $\Psi(F)\left(t_{1} / \equiv, \cdots, t_{k} / \equiv\right)=\Phi(F)\left(t_{1}, \cdots, t_{k}\right) / \equiv$, for every $t_{1}, \cdots, t_{k} \in T$ and every $k$-ary connective $F$. Notice the fact that the function $\Psi(F)$ is well-defined because of $\mathrm{C} 2$.

(c) $E=\{t / \equiv \mid t \in D\}$

By C3, $t / \equiv \in E \Leftrightarrow t \in D$ for every $t \in T$. Let $h$ be the function which maps each $t \in T$ to $t / \equiv \in U$. Since $h$ is a surjective homomorphism of $\mathscr{L}$ into $\mathscr{L} \mid \equiv$, by Lemma 2, we obtain the following

Proposition 5. Let $\mathscr{L}$ be a logic and $\equiv$ a congruence relation on it. Then

$$
\begin{gathered}
W(\mathscr{L} \mid \equiv)=W(\mathscr{L}) . \\
-248-
\end{gathered}
$$


2.52. Let $\mathscr{L}=(T, \Phi, D)$ be a logic and $S$ an $\mathscr{L}$-subset.

We let $\mathscr{L} \uparrow S$ be the logic $(U, \Psi, E)$ defined by (a), (b) and (c):

(a) $U=S$

(b) $\Psi(F)\left(u_{1}, \cdots, u_{k}\right)=\Phi(F)\left(u_{1}, \cdots, u_{k}\right)$, for every $u_{1}, \cdots, u_{k} \in U$ and every $k$-ary connective $F$. Notice that the function $\Psi(F)$ maps $U^{k}$ into $U$ because of the property of $S$.

(c) $E=D \cap S$.

Let $h$ be the function which maps each $u \in U$ to $u \in T$. Since $h$ is a homomorphism of $\mathscr{L} \uparrow S$ into $\mathscr{L}$, by Lemma 2 , we obtain the following

Proposition 6. Let $\mathscr{L}$ be a logic and $S$ an $\mathscr{L}$-subset. Then

$$
W(\mathscr{L} \uparrow S) \supseteq W(\mathscr{L})
$$

2.53. Let $\mathscr{L}=(T, \Phi, D)$ be a logic and $U$ a set satisfying

$$
\operatorname{Card}(U) \geq \operatorname{Card}(T) \text {. }
$$

Then, utilizing a function $h$ mapping $U$ onto $T$ which exists by the assumption, we let $\mathfrak{M}=(U, \Psi, E)$ be such a logic that:

(a) $U$ is given.

(b) $h\left(\Psi(F)\left(u_{1}, \cdots, u_{k}\right)\right)=\Phi(F)\left(h\left(u_{1}\right), \cdots, h\left(u_{k}\right)\right)$, for every $u_{1}, \cdots, u_{k} \in U$ and every $k$-ary connective $F$. Notice that such a function $\Psi(F)$ exists since $h$ is surjective.

(c) $E=\{u \in U \mid h(u) \in D\}$.

Since $h$ is a surjective homomorphism of $2 \llbracket 6$ into $\mathscr{L}$, by Lemma 2, we obtain the fact that

$$
\boldsymbol{W}(\mathfrak{M})=\boldsymbol{W}(\mathscr{L}) .
$$

Thus we have proved the following proposition essentially due to Kalicki [1] in which valid formulas and only finite logics are treated.

Proposition 7. Let $\mathscr{L}=(T, \Phi, D)$ be a logic and $U$ a set satisfying

$$
\operatorname{Card}(U) \geq \operatorname{Card}(T) \text {. }
$$

Then there is a logic $\mathfrak{M}=(U, \Psi, E)$ such that

$$
\boldsymbol{W}(\mathfrak{M})=\boldsymbol{W}(\mathscr{L}) \text {. }
$$

By Proposition 7, for each logic we can arbitrarily increase the number of truth-values without altering the valid sequents.

\section{§3. Characterization of the sets of all the valid sequents}

3.1. The main purpose of this section is to prove Theorem 8 below which characterizes those sets which are exactly the sets of all the valid sequents in some logics. To state Theorem 8 , we shall first list properties V1-V6 concerning a set $W$ of sequents.

In what follows, $\Gamma, \Theta, A, A, \Gamma_{0}, \Gamma_{1}, \cdots$ shall stand for finite sequences of zero or more formulas. 
V1. The empty sequent

does not belong to $W$.

V2. For every formula $A$, the basic sequent

$$
A \rightarrow A
$$

belongs to $W$.

V3. Suppose that every formula occurring in $\Gamma$ and $\Theta$ also occurs in $\Delta$ and $\Lambda$, respectively. If the sequent

$$
\Gamma \rightarrow \Theta
$$

belongs to $W$, then so does the sequent

$$
\Delta \rightarrow A \text {. }
$$

V4. If two sequents

$$
\Gamma \rightarrow \Theta, A \quad \text { and } \quad A, \Gamma \rightarrow \Theta
$$

belong to $\boldsymbol{W}$, then so does the sequent

$$
\Gamma \rightarrow \Theta .
$$

V5. If a sequent

$$
\Gamma(p) \rightarrow \Theta(p)
$$

belongs to $W$, then so does the result

$$
\Gamma(A) \rightarrow \Theta(A)
$$

of substitution of a formula $A$ for a variable $p$.

V6. Suppose that $\Gamma, \Theta$ and $\Delta, A$ contain no variable in common. If the sequent

$$
\Gamma, \Delta \rightarrow \Theta, A
$$

belongs to $W$, then so does at least one of the sequents

$$
\Gamma \rightarrow \Theta \text { and } \Delta \rightarrow A \text {. }
$$

Then the main theorem of this section runs as follows:

Theorem 8. Let be given a set $\boldsymbol{W}$ of sequents. Then there is a logic $\mathscr{L}$ such that

$$
\boldsymbol{W}=\boldsymbol{W}(\mathscr{L})
$$

iff the properties V1-V6 hold.

3.2. Proof of 'only IF' Part. Let be given a set $W$ of sequents and a logic $\mathscr{L}=(T, \Phi, D)$ and assume that

$$
\begin{gathered}
W=W(\mathscr{L}) \\
-250-
\end{gathered}
$$


Then V1 holds since $T$ is not empty, and V2, V3 and V4 clearly hold. V5 follows from Proposition 1.

With a view to establishing $\mathrm{V} 6$, let $\Gamma, \Theta$ and $\Delta, A$ contain no variable in common, and suppose that neither of the sequents

$$
\Gamma \rightarrow \Theta \quad \text { and } \quad \Delta \rightarrow A
$$

belongs to $W$. Let $p_{1}, \cdots, p_{n}$ and $q_{1}, \cdots, q_{m}$ be all the distinct variables occurring in $\Gamma, \Theta$ and $\Delta, A$, respectively. Then $p_{1}, \cdots, p_{n}, q_{1}, \cdots, q_{m}$ are mutually distinct. Since neither sequent is valid in $\mathscr{L}$, there are elements $t_{1}, \cdots, t_{n}$ and $s_{1}, \cdots, s_{m}$ of $T$ such that those sequents are false in $\mathscr{L}$ at $\left(t_{1}, \cdots, t_{n} / p_{1}, \cdots, p_{n}\right)$ and $\left(s_{1}, \cdots, s_{m} / q_{1}, \cdots\right.$, $\left.q_{m}\right)$, respectively. Then, since the sequent

$$
\Gamma, \Delta \rightarrow \Theta, A
$$

is false at $\left(t_{1}, \cdots, t_{n}, s_{1}, \cdots, s_{m} / p_{1}, \cdots, p_{n}, q_{1}, \cdots, q_{m}\right)$ in $\mathscr{L}$, it is not valid in $\mathscr{L}$, hence it does not belong to $W$. Thus we have proved V6.

3.3. Before the proof of the converse, we shall state two lemmas.

Lemma 9. Let $\boldsymbol{W}$ be a set of sequents satisfying V1, V3, V5 and V6. Then, there are sets $\boldsymbol{A}$ and $\boldsymbol{B}$ of formulas satisfying (1) and (2):

(1) If a sequent $\sigma\left(p_{1}, \cdots, p_{n}\right)$ containing only the distinct variables $p_{1}, \cdots, p_{n}$ does not belong to $W$, then there are formulas $C_{1}, \cdots, C_{n}$ such that each formula occurring in the antecedent of the sequent $\sigma\left(C_{1}, \cdots, C_{n}\right)$ belongs to $\boldsymbol{A}$ while each occurring in the succedent to $B$, where $\sigma\left(C_{1}, \cdots, C_{n}\right)$ is the result of substitution of $C_{1}, \cdots, C_{n}$ for $p_{1}, \cdots, p_{n}$ throughout $\sigma\left(p_{1}, \cdots, p_{n}\right)$.

(2) If $\sigma$ is a sequent whose antecedent and succedent consist solely of those formulas which belong to $\boldsymbol{A}$ and $\boldsymbol{B}$ respectively, then $\sigma$ does not belong to $W$.

To formulate Lemma 10 , is required the notion of complete consistent systems in a set of sequents. A set $\boldsymbol{D}$ of formulas is a complete consistent system in a set $W$ of sequents iff each such sequent $\sigma$ does not belong to $W$ that every formula occurring in the antecedent of $\sigma$ belongs to $D$ while every one occurring in the succedent does not.

Lemma 10 (Maehara [2] ). Let $\boldsymbol{W}$ be a set of sequents satisfying V2, V3 and V4, and $\boldsymbol{A}$ and $\boldsymbol{B}$ sets of formulas satisfying (2). Then there is a set $\boldsymbol{D}$ of formulas satisfying (3) and (4):

(3) $\boldsymbol{A} \subseteq \boldsymbol{D}$ and $\boldsymbol{B} \cap \boldsymbol{D}=\boldsymbol{\phi}$.

(4) $\boldsymbol{D}$ is a complete consistent system in $W$.

3.31. Proof of 'IF' Part of Theorem 8. Suppose that we have proved Lemmas 9 and 10. Let be given a set $W$ of sequents, and assume that the properties V1-V6 hold. First take sets $\boldsymbol{A}$ and $\boldsymbol{B}$ of formulas satisfying (1) and (2) by Lemma 9 , and next take a set $\boldsymbol{D}$ of formulas satisfying (3) and (4) by Lemma 10. Then making use of V5, (4) and (1), (3), a sequent $\sigma\left(p_{1}, \cdots, p_{n}\right)$ containing only 
the distinct variables $p_{1}, \cdots, p_{n}$ belongs to $W$ iff for every formulas $C_{1}, \cdots, C_{n}$ some formulas occurring in the succedent of the sequent $\sigma\left(C_{1}, \cdots, C_{n}\right)$ belong to $D$ whenever all occurring in the antecedent do, where $\sigma\left(C_{1}, \cdots, C_{n}\right)$ is the result of substitution of $C_{1}, \cdots, C_{n}$ for $p_{1}, \cdots, p_{n}$ throughout $\sigma\left(p_{1}, \cdots, p_{n}\right)$. Hence by Proposition 4 , we obtain the fact that

$$
W=W(\mathscr{L}(D))
$$

3.32. Proof of Lemma 9. Let be given a set $W$ of sequents satisfying V1, V3, V5 and V6. Let $\sigma_{0}, \sigma_{1}, \cdots, \sigma_{i}, \cdots$ be all those sequents which do not belong to $W$.

We shall define, by induction, for each non-negative integer $i$ a sequent

$$
\tau_{i}: \Delta_{i} \rightarrow \Lambda_{i}
$$

so that

$(5)_{i} \tau_{i}$ does not belong to $W$.

First, we define $\tau_{0}$ to be the empty sequent

$$
\rightarrow \text {. }
$$

Then $(5)_{0}$, holds by V1. Next, suppose that $\tau_{i}$ has been so defined that $(5)_{i}$ holds. In view of $\mathrm{V} 5$ by suitable renaming of variables we obtain a sequent

$$
\boldsymbol{o}_{i}^{\prime}: \quad \Gamma_{i} \rightarrow \Theta_{i}
$$

satisfying (6), (7) and (8) below, and we define $\tau_{i+1}$ to be the sequent

$$
\Gamma_{i}, \Delta_{i} \rightarrow \Theta_{i}, \Lambda_{i} \text {. }
$$

(6) $\sigma_{i}{ }^{\prime}$ is a result of substitution of formulas, variables in fact, for variables throughout $\sigma_{i}$.

(7) $\sigma_{i}^{\prime}$ does not belong to $W$.

(8) $\Gamma_{i}, \Theta_{i}$ and $\Delta_{i}, A_{i}$ contain no variable in common.

Then (5) $)_{i+1}$ holds by V6. Thus, we have defined for each non-negative integer $i$ a sequent

$$
\tau_{i}: \Delta_{i} \rightarrow \Lambda_{i}
$$

so that $(5)_{i}$ holds. Moreover, every formula occurring in $\Delta_{i}$ or $\Lambda_{i}$ also occurs in $\Delta_{i+1}$ or $\Lambda_{i+1}$, respectively.

Then, by V3 it suffices for the present purpose to take the set of all the formulas occurring in $\Delta_{0}, \Delta_{1}, \cdots, \Delta_{i}, \cdots$ or $\Lambda_{0}, A_{1}, \cdots, A_{i}, \cdots$ as $A$ or $B$, respectively.

3.33. Lemma 10 is Theorem 2 of Maehara [2], and we omit the proof.

3.4. As a corollary to the proof of Theorem 8, we obtain the following

Proposition 11. For every logic $\mathscr{L}$ there is a complete consistent system $D$ in $W(\mathscr{L})$ such that 


$$
W(\mathscr{L}(D))=W(\mathscr{L}) .
$$

3.5. Note in passing the following proposition in which a non-empty family of logics is concerned, while in Theorem 8 a logic is.

Proposition 12. Let be given a set $W$ of sequents. Then there is a non-empty family $\left\{\mathscr{L}_{\kappa} \mid \kappa \in \boldsymbol{K}\right\}$ of logics such that

$$
W=\bigcap_{\kappa \in K} W\left(\mathscr{L}_{\kappa}\right)
$$

iff the properties V1-V5 hold.

The above proposition is proved similarly to the proof of Theorem 8. For the proof of 'if' part, it suffices to take the family of all $\mathscr{L}(D)$ 's for which $D$ is a complete consistent system in $W$ as the non-empty family of logics.

\section{§4. Regularity and finite logics}

4.1. Preparatory to defining the notion of regularity of logics, we shall define the relation $\equiv_{s}$ for each logic $\mathscr{L}$.

Let $\mathscr{L}=(T, \Phi, D)$ be a logic, and suppose that $p, q_{1}, q_{2}, \cdots, q_{m}, \cdots$ are distinct variables. For each elements $r$ and $s$ of $T$, let $r \equiv_{s} s$ mean that

$$
A^{\Phi}\left[r, t_{1}, \cdots, t_{m}\right] \in D \Leftrightarrow A^{\Phi}\left[s, t_{1}, \cdots, t_{m}\right] \in D,
$$

for every formula $A\left(p, q_{1}, \cdots, q_{m}\right)$ containing only the variables shown, every elements $t_{1}, \cdots, t_{m}$ of $T$ and for every non-negative integer $m$. Then $\equiv_{f}$ is a congruence relation on $\mathscr{L}$, hence we can construct the logic $\mathscr{L} \mid \equiv_{\boldsymbol{\rho}}$. Moreover, if $\equiv$ is a congruence relation on $\mathscr{L}$, then $r \equiv_{s} s$ whenever $r \equiv s$.

LEMma 13. Let $\mathscr{L}$ be a logic and $n$ a non-negative integer. If

then

$$
\operatorname{Card}\left(\mathscr{L} \mid \equiv_{\mathscr{s}}\right) \geq n \text {, }
$$

$$
(\mathfrak{M})[\boldsymbol{W}(\mathfrak{M}) \subseteq W(\mathscr{L}) \Rightarrow \operatorname{Card}(\mathfrak{M}) \geq n],
$$

where $\mathfrak{M}$ ranges over arbitrary logics.

Proof. Let be given logics $\mathscr{L}=(T, \Phi, D)$ and $\mathfrak{M}=(U, \Psi, E)$ and a nonnegative integer $n$, and assume that

$$
\operatorname{Card}\left(\mathscr{L} \mid \equiv_{s}\right) \geq n \quad \text { and } \quad W(\mathfrak{R}) \subseteq W(\mathscr{L}) .
$$

Since there are at least $n$ elements of $T$ which are not mutually equivalent in the sense of $\equiv_{\infty}$, for some integer $m$ not smaller than $n$ there are elements $t_{1}, \cdots, t_{n}$, $t_{n+1}, \cdots, t_{m}$ of $T$ such that for each distinct elements $i$ and $j$ of $\{1, \cdots, n\}$ there is a formula $A_{i j}\left(p, q_{1}, \cdots, q_{m}\right)$ containing only the distinct variables $p, q_{1}, \cdots, q_{m}$ for which either

or

$$
Q(i, j): A_{i j}^{\Phi}\left[t_{i}, t_{1}, \cdots, t_{m}\right] \in D \quad \text { and } \quad A_{i j}{ }^{\Phi}\left[t_{j}, t_{1}, \cdots, t_{m}\right] \notin D,
$$

$$
R(i, j): \quad A_{i j}{ }^{\Phi}\left[t_{i}, t_{1}, \cdots, t_{m}\right] \notin D \quad \text { and } \quad A_{i j}{ }^{\Phi}\left[t_{j}, t_{1}, \cdots, t_{m}\right] \in D
$$


Then take a sequent $\sigma\left(q_{1}, \cdots, q_{m}\right)$ whose antecedent consists of:

and those $A_{i j}\left(q_{i}, q_{1}, \cdots, q_{m}\right)$ 's for which $Q(i, j)$ holds, those $A_{i j}\left(q_{j}, q_{1}, \cdots, q_{m}\right)$ 's for which $R(i, j)$ holds, while whose succedent consists of:

$$
\begin{aligned}
& \text { those } A_{i j}\left(q_{j}, q_{1}, \cdots, q_{m}\right) \text { 's for which } Q(i, j) \text { holds, } \\
& \text { and } \\
& \text { those } A_{i j}\left(q_{i}, q_{1}, \cdots, q_{m}\right) \text { 's for which } R(i, j) \text { holds. }
\end{aligned}
$$

Since $\sigma\left[t_{1}, \cdots, t_{m}\right]$ is false in $\mathscr{L}, \sigma\left(q_{1}, \cdots, q_{m}\right)$ does not belong to $W(\mathscr{L})$. Therefore $\boldsymbol{\sigma}\left(q_{1}, \cdots, q_{m}\right)$ does not belong also to $W(\mathfrak{R})$. Hence there are elements $u_{1}, \cdots, u_{m}$ of $U$ such that $\sigma\left[u_{1}, \cdots, u_{m}\right]$ is false in $\mathfrak{M}$.

Then, we claim that

(9) $u_{1}, \cdots, u_{n}$ are mutually distinct, from which follows the fact that

$$
\operatorname{Card}(\mathfrak{M}) \geq n .
$$

For the proof of (9), let $i$ and $j$ be distinct elements of $\{1, \cdots, n\}$. Then either $Q(i, j)$ or $R(i, j)$ holds. In the former case

$$
A_{i j} \Psi\left[u_{i}, u_{1}, \cdots, u_{m}\right] \in E \quad \text { and } \quad A_{i j} \Psi\left[u_{j}, u_{1}, \cdots, u_{m}\right] \notin E,
$$

while in the latter case

$$
A_{i j} \Psi\left[u_{i}, u_{1}, \cdots, u_{m}\right] \notin E \quad \text { and } \quad A_{i j} \Psi\left[u_{j}, u_{1}, \cdots, u_{m}\right] \in E .
$$

Hence $u_{i} \neq u_{j}$ in either case.

Corollary 14. Let $\mathscr{L}$ be a finite logic and $\mathfrak{M}$ any logic, and suppose that

$$
\boldsymbol{W}(\mathscr{L}) \subseteq \boldsymbol{W}(\mathfrak{M})
$$

Then there is a finite logic $\mathfrak{M}^{\prime}$ such that

$$
\boldsymbol{W}\left(\mathfrak{M}^{\prime}\right)=W(\mathfrak{M})
$$

Proof. First, by Proposition 5 we have

$$
W(\mathfrak{M} / \equiv \mathfrak{m})=W(\mathfrak{M})
$$

Next, we claim that

(10) $\operatorname{Card}\left(\mathfrak{M} / \equiv_{\mathfrak{m}}\right) \leq \operatorname{Card}(\mathscr{L})$,

from which follows the fact that the logic $\mathfrak{M} / \equiv_{\mathfrak{M}}$ is finite. Hence, it suffices for the present purpose to take $\mathfrak{M} / \equiv_{\mathfrak{m}}$ as $\mathfrak{M}^{\prime}$.

For the proof of (10), suppose that (10) does not hold.

Then, since

$$
\begin{gathered}
\operatorname{Card}(\mathfrak{M} / \equiv \mathbb{R}) \geq \operatorname{Card}(\mathscr{L})+1 \quad \text { and } \quad W(\mathscr{L}) \subseteq W(\mathfrak{M}), \\
-\mathbf{2 5 4}-
\end{gathered}
$$


by Lemma 13 we have

$$
\operatorname{Card}(\mathscr{L}) \geq \operatorname{Card}(\mathscr{L})+1,
$$

which is contradictory. Hence (10) has been proved.

4.2. Now, we have arrived at the stage where the notion of regularity of logics is defined.

A logic $\mathscr{L}$ is regular iff $r=s$ whenever $r \equiv_{s} s$.

Notice that a finite logic $\mathscr{L}$ is regular iff

$$
\operatorname{Card}\left(\mathscr{L} \mid \equiv_{s}\right) \geq \operatorname{Card}(\mathscr{L}) \text {. }
$$

We have the following proposition for arbitrary logics.

Proposition 15. For every logic $\mathscr{L}$, the logic $\mathscr{L} / \equiv_{\mathscr{S}}$ is regular.

Proof. Let $\mathscr{L}=(T, \Phi, D)$ and $\mathscr{L} / \equiv_{\mathscr{s}}=\mathfrak{M}=(U, \Psi, E)$. Suppose that $v$ and $w$ are distinct elements of $U$. Then there are elements $r$ and $s$ of $T$ for which $v=r / \equiv_{s}$, $w=s / \equiv_{s}$ and it does not hold that $r \equiv_{s} s$. Take a formula $A\left(p, q_{1}, \cdots, q_{m}\right)$ containing only the distinct variables $p, q_{1}, \cdots, q_{m}$, and elements $t_{1}, \cdots, t_{m}$ of $T$ for which it is not the case that

$$
A^{\Phi}\left[r, t_{1}, \cdots, t_{m}\right] \in D \Leftrightarrow A^{\Phi}\left[s, t_{1}, \cdots, t_{m}\right] \in D .
$$

Then, since the function which maps each $t \in T$ to $t / \equiv_{f} \in U$ is a (surjective) homomorphism of $\mathscr{L}$ into $\mathfrak{M}$, by Proposition 3 it is not the case that

$$
A^{\Psi}\left[v, t_{\mathbf{1}} / \equiv_{s}, \cdots, t_{m} / \equiv_{s}\right] \in E \Leftrightarrow A^{\Psi}\left[w, t_{1} / \equiv_{s}, \cdots, t_{m} / \equiv_{s}\right] \in E .
$$

Therefore it does not hold that $v \equiv_{\mathfrak{m}} u$. Hence $v=w$ whenever $v \equiv_{\mathfrak{n}} w$, that is, $\mathscr{L} \mid \equiv_{\boldsymbol{s}}=\mathfrak{M}$ is regular.

Making use of Proposition 5, we have the following

Corollary 16. For every logic $\mathscr{L}$, there is a regular logic $\mathscr{L}^{\prime}$ such that

$$
W\left(\mathscr{L}^{\prime}\right)=W(\mathscr{L})
$$

If $\mathscr{L}$ is funite, we can take $\mathscr{L}^{\prime}$ so that it is also finite.

4.3. The equivalency of (11) and (12) of the following theorem, which is almost clear after the previous consideration, characterizes those finite logics for which we cannot decrease the number of truth-values without altering the valid sequents.

THeоRем 17. If $\mathscr{L}$ is a finite logic, then the following assertions (11), (12) and (13) are equivalent, where $\mathfrak{M}$ ranges over finite or arbitrary logics:

(11) $\mathscr{L}$ is regular.

(12) $(\mathfrak{M})[W(\mathfrak{M})=W(\mathscr{L}) \Rightarrow \operatorname{Card}(\mathfrak{M}) \geq \operatorname{Card}(\mathscr{L})]$.

(13) $(\mathfrak{M})[W(\mathfrak{M}) \subseteq W(\mathscr{L}) \Rightarrow \operatorname{Card}(\mathfrak{M}) \geq \operatorname{Card}(\mathscr{L})]$.

Proof. Let be given a finite logic $\mathscr{L}$.

$(13) \Rightarrow(12)$. Evident. 
$(12) \Rightarrow(11)$. Assume (12). Then, since by Proposition 5

$$
W\left(\mathscr{L} \mid \equiv_{\mathscr{s}}\right)=W(\mathscr{L}),
$$

we have

$$
\operatorname{Card}\left(\mathscr{L} \mid \equiv_{\mathscr{s}}\right) \geq \operatorname{Card}(\mathscr{L})
$$

that is, $\mathscr{L}$ is regular.

(11) $\Rightarrow(13)$. Assume (11). Then, since

$$
\operatorname{Card}\left(\mathscr{L} \mid \equiv_{\mathscr{f}}\right) \geq \operatorname{Card}(\mathscr{L}),
$$

we have by Lemma 13

$$
(\mathfrak{M})[W(\mathfrak{M}) \subseteq W(\mathscr{L}) \Rightarrow \operatorname{Card}(\mathfrak{M}) \geq \operatorname{Card}(\mathscr{L})] .
$$

4.4. The following theorem is efficient in determining for a finite logic $\mathscr{L}$ such a logic $\mathfrak{M}$ that

$$
W(\mathscr{L}) \subseteq W(\mathfrak{R})
$$

THEOREM 18. Let $\mathscr{L}$ be a finite logic and $\mathfrak{M}$ a regular, finite one. If

$$
\boldsymbol{W}(\mathscr{L}) \subseteq W(\mathfrak{R}),
$$

then there is an $\mathscr{L}$-subset $S$ such that

$$
\operatorname{Card}(S) \geq \operatorname{Card}(\mathfrak{M}) \quad \text { and } \quad W(\mathscr{L} \uparrow S)=W(\mathfrak{M}) .
$$

4.41. Before the proof of Theorem 18, we shall state two lemmas.

LeMma 19. Let $\mathscr{L}=(T, \Phi, D)$ be a finite logic and $\mathfrak{N}=(U, \Psi, E)$ any logic such that

$$
W(\mathscr{L}) \subseteq W(\mathfrak{M})
$$

Suppose that $u_{1}, \cdots, u_{n}$ are elements of $U$. Then, there are elements $t_{1}, \cdots, t_{n}$ of $T$ satisfying (14):

(14) For every formula $B\left(q_{1}, \cdots, q_{n}\right)$ containing only the distinct variables $q_{1}, \cdots$, $q_{n}$,

$$
B^{\Phi}\left[t_{1}, \cdots, t_{n}\right] \in D \Leftrightarrow B \Psi\left[u_{1}, \cdots, u_{n}\right] \in E .
$$

Lemma 20. Let $\mathscr{L}=(T, \Phi, D)$ be any logic and $\mathfrak{M}=(U, \Psi, E)$ a regular, finite one. Suppose that $u_{1}, \cdots, u_{n}$ are all the elements of $U$ and $t_{1}, \cdots, t_{n}$ elements of $T$ satisfying (14). Then for every formulas $B\left(q_{1}, \cdots, q_{n}\right)$ and $C\left(q_{1}, \cdots, q_{n}\right)$ containing only the distinct variables $q_{1}, \cdots, q_{n}$,

$$
B^{\Phi}\left[t_{1}, \cdots, t_{n}\right]=C^{\Phi}\left[t_{1}, \cdots, t_{n}\right] \Rightarrow B^{\Psi}\left[u_{1}, \cdots, u_{n}\right]=C \Psi\left[u_{1}, \cdots, u_{n}\right] .
$$

4.42. Proof of Theorem 18. Suppose that we have proved Lemmas 19 and 20. Let $\mathscr{L}=(T, \Phi, D), \mathfrak{M}=(U, \Psi, E)$, and let $u_{1}, \cdots, u_{n}$ be all the distinct elements of $U$. Let $q_{1}, \cdots, q_{n}$ be distinct variables, and suppose that

$$
\begin{gathered}
W(\mathscr{L}) \subseteq W(\mathfrak{M}) . \\
-256-
\end{gathered}
$$


Then, take elements $t_{1}, \cdots, t_{n}$ of $T$ satisfying (14) by Lemma 19 and define $S=\left\{B^{\Phi}\left[t_{1}, \cdots, t_{n}\right] \mid B\left(q_{1}, \cdots, q_{n}\right)\right.$ is a formula containing only the variables shown $\}$.

Then $S$ is an $\mathscr{L}$-subset containing $t_{1}, \cdots, t_{n}$. Since $u_{1}, \cdots, u_{n}$ are mutually distinct, so are $t_{1}, \cdots, t_{n}$ by Lemma 20 . Therefore

$$
\operatorname{Card}(S) \geq n=\operatorname{Card}(\mathfrak{M}) \text {. }
$$

Now, we shall define a function $h$ mapping $S$ into $U$ by putting

$$
h\left(B^{\Phi}\left[t_{1}, \cdots, t_{n}\right]\right)=B^{\Psi}\left[u_{1}, \cdots, u_{n}\right],
$$

for each formula $B\left(q_{1}, \cdots, q_{n}\right)$ containing only the variables shown. Notice that the function $h$ is well-defined because of Lemma 20 .

Then, we claim that

(15) $h$ is a surjective homomorphism of $\mathscr{L} \uparrow S$ into $\mathfrak{M}$,

from which together with Lemma 2 follows the fact that

$$
W(\mathscr{L} \uparrow S)=W(\mathfrak{M})
$$

Let us prove (15). First, $h$ maps $S$ onto $U$ since $h\left(t_{1}\right)=u_{1}, \cdots, h\left(t_{n}\right)=u_{n}$. Next, we shall show $\mathrm{H} 1$, that is,

$$
h\left(\Phi(F)\left(s_{1}, \cdots, s_{k}\right)\right)=\Psi(F)\left(h\left(s_{1}\right), \cdots, h\left(s_{k}\right)\right),
$$

for every $s_{1}, \cdots, s_{k} \in S$ and every $k$-ary connective $F$. Take $s_{1}, \cdots, s_{k} \in S$ and a $k$ ary connective $F$. Let $s_{1}=B_{1}{ }^{\Phi}\left[t_{1}, \cdots, t_{n}\right], \cdots, s_{k}=B_{k}{ }^{\Phi}\left[t_{1}, \cdots, t_{n}\right]$ and $B\left(q_{1}, \cdots, q_{n}\right)$ be $F\left(B_{1}\left(q_{1}, \cdots, q_{n}\right), \cdots, B_{k}\left(q_{1}, \cdots, q_{n}\right)\right)$. Then

$$
\begin{aligned}
& h\left(\Phi(F)\left(s_{1}, \cdots, s_{k}\right)\right) \\
= & h\left(\Phi(F)\left(B_{1}{ }^{\Phi}\left[t_{1}, \cdots, t_{n}\right], \cdots, B_{k}{ }^{\Phi}\left[t_{1}, \cdots, t_{n}\right]\right)\right) \\
= & h\left(B^{\Phi}\left[t_{1}, \cdots, t_{n}\right]\right) \\
= & B^{\Psi}\left[u_{1}, \cdots, u_{n}\right] \\
= & \left.\Psi(F)\left(B_{1} \Psi\left[u_{1}, \cdots, u_{n}\right]\right), \cdots, B_{k} \Psi\left[u_{1}, \cdots, u_{n}\right]\right) \\
= & \Psi(F)\left(h\left(B_{1}{ }^{\Phi}\left[t_{1}, \cdots, t_{n}\right]\right), \cdots, h\left(B_{k}{ }^{\Phi}\left[t_{1}, \cdots, t_{n}\right]\right)\right) \\
= & \Psi(F)\left(h\left(s_{1}\right), \cdots, h\left(s_{k}\right)\right) .
\end{aligned}
$$

Lastly, we shall show $\mathrm{H} 2$, that is,

$$
h(s) \in E \Leftrightarrow s \in D \cap S,
$$

for every $s \in S$. Take $s \in S$ and let $s=B^{\Phi}\left[t_{1}, \cdots, t_{n}\right]$. Then

$$
\begin{aligned}
& h(s) \in E \\
\Leftrightarrow & h\left(B^{\Phi}\left[t_{1}, \cdots, t_{n}\right]\right) \in E \\
\Leftrightarrow & B^{\Psi}\left[u_{1}, \cdots, u_{n}\right] \in E \\
\Leftrightarrow & B^{\Phi}\left[t_{1}, \cdots, t_{n}\right] \in D \\
\Leftrightarrow & s \in D \cap S,
\end{aligned}
$$

by (14). Hence we have proved (15).

4.43. Proof of Lemma 19. Suppose not, and take for each $t=\left(t_{1}, \cdots, t_{n}\right)$ 
$\epsilon T_{n}$ a formula $B_{t}\left(q_{1}, \cdots, q_{n}\right)$ containing only the variables shown such that either

or

$$
Q(t): B_{t}^{\Phi}\left[t_{1}, \cdots, t_{n}\right] \in D \quad \text { and } \quad B_{t}{ }^{\Psi}\left[u_{1}, \cdots, u_{n}\right] \notin E
$$

$$
R(t): B_{t}^{\Phi}\left[t_{1}, \cdots, t_{n}\right] \notin D \quad \text { and } \quad B_{t} T\left[u_{1}, \cdots, u_{n}\right] \in E .
$$

Take a sequent $\sigma\left(q_{1}, \cdots, q_{n}\right)$ whose antecedent consists of:

$$
\text { those } B_{t}\left(q_{1}, \cdots, q_{n}\right) \text { 's for which } R(t) \text { holds, }
$$

while whose succedent consists of:

$$
\text { those } B_{t}\left(q_{1}, \cdots, q_{n}\right) \text { 's for which } Q(t) \text { holds. }
$$

Since $\sigma\left[u_{1}, \cdots, u_{n}\right]$ is false in $\mathfrak{M}$,

(16) $\sigma\left(q_{1}, \cdots, q_{n}\right)$ does not belong to $W(\mathfrak{M})$.

On the other hand, we claim that

(17) $\sigma\left(q_{1}, \cdots, q_{n}\right)$ belongs to $W(\mathscr{L})$,

which with (16) contradicts to the assumption.

For the proof of $(17)$, let $t=\left(t_{1}, \cdots, t_{n}\right)$ be an arbitrary element of $T^{n}$. Then either $Q(t)$ or $R(t)$ holds. In the former case the formula $\boldsymbol{B}_{t}\left(q_{1}, \cdots, q_{n}\right)$ occurs in the succedent of $\sigma\left(q_{1}, \cdots, q_{n}\right)$ and $B_{t}{ }^{\Phi}\left[t_{1}, \cdots, t_{n}\right] \in D$, while in the latter case it occurs in the antecedent and $B_{t}^{\Phi}\left[t_{1}, \cdots, t_{n}\right] \notin D$, so $\sigma\left[t_{1}, \cdots, t_{n}\right]$ is true in $\mathscr{L}$ in either case. Hence $\sigma\left(q_{1}, \cdots, q_{n}\right)$ is valid in $\mathscr{L}$.

4.44. Proof of Lemma 20. Assume that $B \Psi\left[u_{1}, \cdots, u_{n}\right] \neq C \Psi\left[u_{1}, \cdots, u_{n}\right]$. Since $\mathfrak{M}$ is regular, there is a formula $A\left(p, q_{1}, \cdots, q_{n}\right)$ containing only the variables shown, where $p$ is a variable distinct from $q_{1}, \cdots, q_{n}$, for which it is not the case that

$$
A^{\Psi}\left[B \Psi\left[u_{1}, \cdots, u_{n}\right], u_{1}, \cdots, u_{n}\right] \in E \Leftrightarrow A^{\Psi}\left[C \Psi\left[u_{1}, \cdots, u_{n}\right], u_{1}, \cdots, u_{n}\right] \in E .
$$

Hence by Proposition 1 and (14), it is not the case that

$$
A^{\Phi}\left[B^{\Phi}\left[t_{1}, \cdots, t_{n}\right], t_{1}, \cdots, t_{n}\right] \in D \Leftrightarrow A^{\Phi}\left[C^{\Phi}\left[t_{1}, \cdots, t_{n}\right], t_{1}, \cdots, t_{n}\right] \in D .
$$

Therefore $B^{\Phi}\left[t_{1}, \cdots, t_{n}\right] \neq C^{\Phi}\left[t_{1}, \cdots, t_{n}\right]$.

4.45. By the following corollary, for a finite logic $\mathscr{L}$ such sets $W(\mathfrak{M})$ of the valid sequents in some logic $\mathfrak{M}$ as

$$
W(\mathfrak{M}) \supseteq W(\mathscr{L})
$$

are determined by $\mathscr{L}$-subsets.

Corollary 21. Let $\mathscr{L}$ be a finite logic and $\mathfrak{M}$ any logic. Then

$$
\boldsymbol{W}(\mathscr{L}) \subseteq W(\mathfrak{M})
$$

iff there is an $\mathscr{L}$-subset $S$ such that

$$
W(\mathscr{L} \uparrow S)=W(\mathfrak{R})
$$

Proof. 'If' part. By Proposition 6. 
'ONLY IF' PART. In view of Corollaries 14 and 16 there is a regular, finite logic $\mathfrak{M}^{\prime}$ such that

$$
W\left(\mathfrak{M} \mathfrak{R}^{\prime}\right)=W(\mathfrak{M}),
$$

by the assumption. So the conclusion follows from Theorem 18.

From Corollary 21 follows

CoRollary 22. Let $\mathscr{L}$ be a finite logic, and suppose that there is no proper $\mathscr{L}$ subset. Then there is no logic $\mathfrak{M}$ such that

$$
W(\mathfrak{M}) \supset W(\mathscr{L}) .
$$

Proof. Let be given a logic $\mathfrak{M}$, and suppose that

$$
W(\mathfrak{M}) \supseteq W(\mathscr{L}) \text {. }
$$

We shall deduce

$$
W(\mathfrak{M})=W(\mathscr{L}) .
$$

By Corollary 21, there is an $\mathscr{L}$-subset $S$ such that

$$
W(\mathscr{L} \uparrow S)=W(\mathfrak{M}) \text {. }
$$

Since $S$ is not a proper $\mathscr{L}$-subset by the assumption, $\mathscr{L} \uparrow S=\mathscr{L}$, and so

$$
W(\mathscr{L})=W(\mathfrak{M}) \text {. }
$$

4.5. Combining Theorem 17 and Corollary 22, we obtain the following theorem which is an addendum of Theorem 17 .

Theorem 23. If $\mathscr{L}$ is a finite logic, then (18) and (19) below are equivalent, where $\mathfrak{M}$ ranges over finite or arbitrary logics:

(18) $\mathscr{L}$ is regular, and there is no proper $\mathscr{L}$-subset.

(19) $(\mathfrak{M})[W(\mathfrak{M}) \supseteq W(\mathscr{L}) \Rightarrow \operatorname{Card}(\mathfrak{M}) \geq \operatorname{Card}(\mathscr{L})]$.

Proof. $\quad(19) \Rightarrow(18)$. Assume (19). Then $\mathscr{L}$ is regular by Theorem 17 . Next, if $S$ is a proper $\mathscr{L}$-subset, then by Proposition 6 we have

$$
W(\mathscr{L}) \uparrow S) \supseteq W(\mathscr{L}) \quad \text { and } \quad \operatorname{Card}(\mathscr{L} \uparrow S)<\operatorname{Card}(\mathscr{L}) \text {, }
$$

contradicting to the assumption, hence no such $S$ exists.

$(18) \Rightarrow(19)$. Assume (18). Take a logic $\mathfrak{M}$, and suppose that

$$
W(\mathfrak{M}) \supseteq W(\mathscr{L}) \text {. }
$$

Then by Corollary 22,

$$
W(\mathfrak{M})=W(\mathscr{L})
$$

Hence by Theorem 17, we have

$$
\operatorname{Card}(\mathfrak{M}) \geq \operatorname{Card}(\mathscr{L}) .
$$

Making use of Theorems 17 and 18 we also obtain the following 
Theorem 24. Let $\mathscr{L}$ be any logic and $\mathfrak{M}$ a regular, finite one. Then

$$
W(\mathscr{L}) \subset W(\mathfrak{M}) \Rightarrow \operatorname{Card}(\mathscr{L})>\operatorname{Card}(\mathfrak{M}) \text {. }
$$

Proof. Assume that

$$
W(\mathscr{L}) \subset W(\mathfrak{M})
$$

Then by Theorem 17, we have

$$
\operatorname{Card}(\mathscr{L}) \geq \operatorname{Card}(\mathfrak{M}) \text {. }
$$

Now, suppose that

$$
\operatorname{Card}(\mathscr{L})=\operatorname{Card}(\mathfrak{M}) \text {. }
$$

Then by Theorem 18 since $\mathscr{L}$ is finite, there is an $\mathscr{L}$-subset $S$ such that

$$
\operatorname{Card}(S) \geq \operatorname{Card}(\mathfrak{M}) \quad \text { and } \quad W(\mathscr{L} \uparrow S)=W(\mathfrak{M}) .
$$

Since

$$
\operatorname{Card}(S) \geq \operatorname{Card}(\mathfrak{M})=\operatorname{Card}(\mathscr{L}) \geq \operatorname{Card}(S),
$$

we have

$$
\operatorname{Card}(S)=\operatorname{Card}(\mathscr{L}),
$$

that is, $S$ is not a proper $\mathscr{L}$-subset, and so $\mathscr{L} \uparrow S=\mathscr{L}$. Hence we have

$$
W(\mathscr{L})=W(\mathfrak{M}),
$$

contradicting to the assumption.

Therefore

$$
\operatorname{Card}(\mathscr{L})>\operatorname{Card}(\mathfrak{M})
$$

\section{References}

[1] J. Kalicki, Note on truth-tables, J. Symbolic Logic, 15 (1950), 174-181.

[2] S. Maehara, A general theory of completeness proofs, this ANNaLs, 3 (1970), 242-256.

[3] A. Tarski, Der Aussagenkalkül und die Topologie, Fund. Math., 31 (1938), 103-134. 